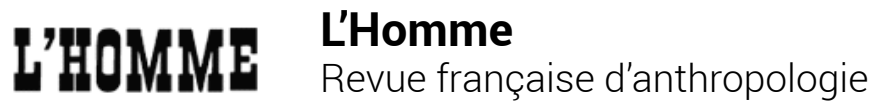

231-232| 2019

Cumulus : Hoarding, Hosting, Hospitality

\section{Christian Bromberger, L'Extraordinaire Destin de Milda Bulle, une pasionaria rouge}

\section{Martine Segalen}

\section{(2) OpenEdition \\ Journals}

\section{Édition électronique}

URL : https://journals.openedition.org/lhomme/35736

DOI : 10.4000/lhomme.35736

ISSN : 1953-8103

Éditeur

Éditions de l'EHESS

\section{Édition imprimée}

Date de publication : 21 novembre 2019

Pagination : 299-302

ISBN : 978-2-7132-2794-3

ISSN : 0439-4216

Référence électronique

Martine Segalen, "Christian Bromberger, L'Extraordinaire Destin de Milda Bulle, une pasionaria rouge», L'Homme [En ligne], 231-232 | 2019, mis en ligne le 21 novembre 2019, consulté le 22 avril 2022. URL http://journals.openedition.org/lhomme/35736 ; DOI : https://doi.org/10.4000/lhomme.35736

Ce document a été généré automatiquement le 22 avril 2022.

(c) École des hautes études en sciences sociales 


\title{
Christian Bromberger, L'Extraordinaire Destin de Milda Bulle, une pasionaria rouge
}

\author{
Martine Segalen
}

\section{RÉFÉRENCE}

Christian Bromberger, L'Extraordinaire Destin de Milda Bulle, une pasionaria rouge. Paris, Créaphis, 2018, 128 p., bibl., gloss., ill.

1 Que venait faire Milda Bulle (prononcer «Boulé»), une jeune Lettonne de 30 ans, dans le nord de l'Iran, théâtre d'un mouvement révolutionnaire qui s'acheva par une éphémère république socialiste soviétique, entre 1920 et 1922 ? Et quelles demandes saugrenues formulait ce professeur français aux conservatrices de la Bibliothèque nationale de Riga, tout interloquées par son intérêt pour une illustre inconnue? La réponse à la seconde question est contenue dans la première. Intrigué, voire fasciné par la découverte plutôt inattendue d'une jeune apparatchik communiste dans le Gilân - une province marginale de l'Iran dont il est le spécialiste reconnu ${ }^{1}-$, Christian Bromberger s'est lancé dans une traque de plusieurs années pour tenter de reconstituer la biographie et la carrière de ce personnage peut-être «extraordinaire » ou, à tout le moins, relativement hors du commun, dont l'auteur se demande si elle incarne une « figure d'exception $»^{2}$.

2 Le résultat de cette enquête est proposé dans un ouvrage de petit format, publié par les éditions Créaphis, réputées pour la qualité esthétique de leurs mises en pages. Ici de nombreuses illustrations, photographies, carte, articles de presse, documents d'archives, permettent de suivre Milda dans ses déplacements, de découvrir les diverses facettes et étapes de sa vie, sans aucun doute exceptionnelles. Mais, au-delà de l'intérêt de la biographie de cette femme singulière, ce qui fait le charme de cet ouvrage est l'histoire de la découverte de Milda, le récit du périple de l'auteur, naviguant entre 
passé soviétique et présent de diverses républiques d'Asie centrale, une mise en abyme très réussie grâce à un style aussi élégant qu'efficace. Le lecteur est ainsi invité à passer du côté de la fabrique du savoir.

3 Dans le premier chapitre, Christian Bromberger s'intéresse aux jeunes années de Milda, née en 1891 et élevée dans un village de la Courlande, une région lettonne située sur la Baltique, dans ce «pays perdu» qui fait alors partie de l'Empire russe. Aînée d'une fratrie de dix enfants, fille d'un instituteur, elle grandit dans un climat révolutionnaire. Mais le mouvement nationaliste dirigé, contre la domination des grands propriétaires terriens dont les manoirs marquent le territoire, s'achève par une répression brutale. Milda se forme, notamment à l'apprentissage des langues étrangères dont elle maîtrisera un grand nombre : l'allemand, le français, le russe, l'anglais et le turc. Ce dévoilement des faits biographiques, le lecteur y accède en même temps que l'auteur de l'ouvrage, qui met constamment en regard le contexte familial, social et politique des jeunes années de Milda, et sa propre expérience de la découverte des traces archivistiques laissées par son héroïne, qui sont autant de signes de la postérité de sa mémoire. Est palpable l'émotion du pudique chercheur lorsqu'il en découvre des indices matériels, remisés dans la soupente de l'école locale: des photographies, une banderole qu'elle porta (Unité de pionniers), etc. Mais ce qui le frappe le plus, ce sont les commentaires de personnalités locales qui, en 2015, ne voient pas en Milda une héroïne, mais un personnage dont la mémoire est attachée à un passé stalinien désormais rejeté. Cet intérêt d'un chercheur français semble ainsi bien incongru: ignore-t-il donc l'histoire chahutée de la Lettonie (première indépendance 1919-1940, envahissement par les Russes, occupation par les nazis jusqu'en 1944, puis annexion en tant que République socialiste soviétique par les Russes avant de retrouver l'indépendance en 1991)?

Dans le deuxième chapitre, Milda nous entraîne dans son périple en Asie centrale avec son époux, Janis Bulle, un poète engagé aux côtés du parti social-démocrate (qui deviendra le parti bolchevik). Ce sont des années de guerre civile très violente dont Christian Bromberger, pour le plus grand profit des lecteurs peu familiers de cette histoire, retrace en détail la chronologie. Nous suivons Milda et Janis depuis Pétrograd (l'ancienne Saint-Pétersbourg), jusque dans diverses villes du Caucase, où se développe son militantisme, en dépit de la mort de son époux. Au cours des années 1918-1919, elle participe, les armes à la main, parfois à cheval, à de féroces combats contre les "Blancs» dans la région, au sein de l'une des armées rouges: la documentation historique a retenu ses actes de bravoure qui lui valurent, dix ans plus tard, d'être décorée de l'Ordre du drapeau rouge, mais qui risquèrent de lui être fatals lorsqu'elle fut atteinte par l'épidémie de typhus. Après son second mariage avec Aboukov, un beau musulman kabarde, Milda se retrouve à Astrakhan, au bord de la mer Caspienne, puis à Bakou devenue, après d'âpres batailles, la capitale de la République socialiste soviétique d'Azerbaïdjan. C'est alors que le couple est envoyé en Iran, au sud de la mer Caspienne, pour tenter de donner une nouvelle orientation à une révolution rurale commencée en 1915. Leur action politique et militaire y fut finalement de courte durée, les Iraniens en révolte considérant que les Bolcheviks, animés par un idéal internationaliste, ne prenaient pas assez en compte leurs coutumes et leur religion. Déchirant le rideau de l'objectivité de la science historique, Christian Bromberger accompagne le lecteur du passé bolchevik au présent de l'enquête, en livrant une description ethnographique de la ville dans laquelle il trouvera les archives les plus complètes sur l'itinéraire de Milda. Le voici à Oufa, capitale de la République de Bachkirie, devenue Bachkortostan en 1990, 
dans l'est de la Russie européenne, à plus de $1500 \mathrm{~km}$ de Moscou. Il y observe les traces du passé soviétique, mais aussi le renouveau du sentiment religieux; il décrit avec humour une manifestation folklorique organisée par les clubs de retraités, originaires des différentes régions de Bachkirie. Ici encore, incompréhension culturelle : pourquoi s'intéresser à Milda et non pas aux «revendications ethniques en Corse, au Pays basque » (p. 32), lui demande-t-on. Décidément, Christian Bromberger est le Huron des Bachkirs!

5 Avant de séjourner à Oufa, après leur passage en Iran, le couple Aboukov est envoyé au nord de Moscou pour mater les divers mouvements insurrectionnels contre le régime bolchevik dont la célèbre révolte des marins de Kronstadt, en 1921. Ils retournent ensuite à Moscou, où tous deux sont admis à l'Académie militaire. Christian Bromberger relate cette période de formation dans le troisième chapitre, puis examine les multiples activités militantes que Milda, à la fin des années 1920, mène au sein du Commissariat du peuple aux affaires étrangères (NKID) - elle y a accès en raison de sa connaissance des langues. Quant à Aboukov, il sera affecté au Commerce intérieur, dans une période, nous rappelle l'auteur, de situation alimentaire particulièrement dramatique. Milda gravit les échelons politiques, devient déléguée du Komintern pour diverses missions. Au cours de ces années, elle rédige de nombreux textes sur la condition féminine, articles, brochures dans lesquelles elle fustige vigoureusement la condition des femmes dans les pays occidentaux autant qu'elle vante celle des femmes en URSS.

6 Dans le quatrième chapitre, le lecteur suit l'action du couple nommé en Bachkirie en 1933. Aboukov y est en charge des sovkhozes, question politique majeure en raison de l'échec de la collectivisation des terres, attribué aux koulaks, « voleurs » et « contrerévolutionnaires ». Milda, elle, devient un véritable apparatchik s'intéressant, dans le cadre des structures politiques, d'abord à la place des femmes dans l'agriculture, puis au développement des activités culturelles, militant activement pour la création de théâtres au sein des kolkhozes, de cinémas, de clubs sportifs, comme d'un opéra à Oufa.

7 L'empathie que l'auteur a manifestée tout au long de l'ouvrage pour son héroïne est particulièrement vive lorsqu'il s'agit, dans le chapitre $V$, de conter « la fin tragique » de Milda et Aboukov, victimes, après d'autres, de dénonciations et de purges. Dans l'analyse du dossier contenant les interrogatoires et leurs procès-verbaux, et en prenant la distance critique qu'il convient face à ces documents, Christian Bromberger partage la souffrance de Milda face aux faux aveux qui lui sont imputés, tout en éclairant la mécanique soviétique de répression qui conduit à sa condamnation à mort : après un procès stalinien, elle fut fusillée en même temps que son époux, en juillet 1938. Sous le récit circonstancié de ces événements, perce l'émotion du chercheur qui réapparait, se met en scène pour évoquer le moment où l'archiviste lui remet le dossier d'instruction de Milda, "après avoir vérifié si celui-ci n'est pas toujours classé "secret" » (p. 95).

Comment donc qualifier cette femme à la trajectoire singulière et, si elle est, comme l'indique le titre du chapitre suivant, une "figure d'exception", pourquoi est-elle tombée dans l'oubli ? Pourquoi l'Histoire n'a-t-elle pas retenu son nom, aux côtés d'autres figures féminines de la révolution russe, telles Rosa Luxembourg, Inessa Armand ou Alexandra Kollontaï? C'est l'objet des courts chapitres VI et VII, dans lesquels Christian Bromberger s'interroge sur la personnalité de Milda, tour à tour femme de courage, victime, mais aussi bourreau. Au fil des pages, l'auteur a aussi 
cherché à élucider les causes de cet oubli relatif. Au cours de la période soviétique de la Lettonie, sa mémoire est restée vivante : une statue en bronze fut édifiée qui remplaça celle de Pierre le Grand ; elle trône encore au centre de la capitale lettonne. Sa mère qui lui survécut obtint, en 1956, sa réhabilitation. À la notoriété, succéda, avec l'indépendance, une période de "démildaisation» qui explique l'étonnement des interlocuteurs de Christian Bromberger.

Figure d'exception? À la marge, Milda en coche toutes les cases, tout d'abord en raison de son sexe dont elle transgresse les normes : elle se lance dans le militantisme; elle est une figure combattante; elle arbore une hexis corporelle relativement masculinisée, comme on le découvre sur les photographies (uniforme militaire, cheveux courts). Dans le domaine privé, ensuite, elle aura deux époux dont, en secondes noces, un homme plus jeune qu'elle de sept années, issu de l'aristocratie et musulman; elle n'a pas d'enfant (est-ce volontaire? on ne le sait). Une femme autoritaire, exigeante, qui a été au bout de ses convictions, vivant un idéal de pureté et dont on peut penser que l'engagement est l'une des possibles expressions de la religiosité, telle a été Milda.

En définitive, L'Extraordinaire Destin de Milda Bulle relève d'un genre mixte, associant à la reconstruction biographique un retour constamment réflexif sur l'enquête en cours. L'ouvrage marie de façon heureuse l'interdisciplinarité entre ethnologie, mémoire et histoire. Il relève aussi d'une quête archéologique lorsque le chercheur rencontre l'objet, le document qui donne vie à un personnage relativement fantasmé. De nombreux excursus historiques sont particulièrement bienvenus, qui permettent de situer des parcours individuels au sein d'un cadre social et politique plus vaste, qu'il s'agisse des guerres civiles d'Asie centrale des années 1920, des révoltes blanches, des soubresauts politiques des différents régimes communistes, comme de la situation des femmes bolcheviks, ou des pratiques culturelles de la République de Bachkirie. Si l'on peut continuer de débattre de la nature de sa trajectoire, Milda Bulle est sortie de la fosse commune du temps grâce à cet ouvrage.

\section{NOTES}

1. Cf. Christian Bromberger, Un autre Iran. Un ethnologue au Gilân, Paris, Armand Colin, 2013.

2. Cf. Christian Bromberger \& Émir Mahieddin, eds, Ethnologie française, 2016, $3: 12$ figures d'exception, Paris, Presses universitaires de France. 